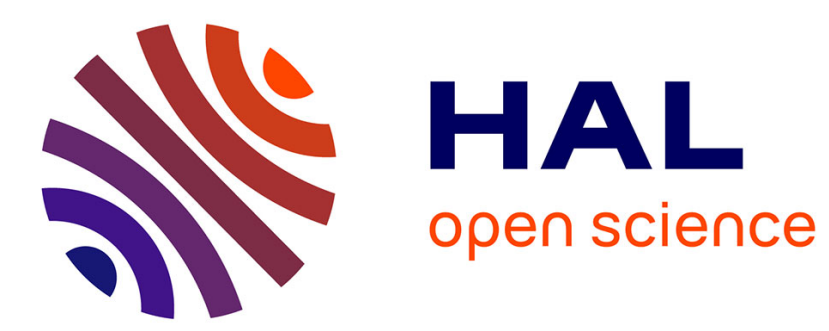

\title{
Excitation Symmetry Analysis Method (ESAM) for calculation of higher order nonlinearities
}

\author{
Serge Dos Santos, Camille Plag
}

\section{To cite this version:}

Serge Dos Santos, Camille Plag. Excitation Symmetry Analysis Method (ESAM) for calculation of higher order nonlinearities. International Journal of Non-Linear Mechanics, 2008, 43 (3), pp.164. 10.1016/j.ijnonlinmec.2007.12.014 . hal-00501773

\section{HAL Id: hal-00501773 \\ https://hal.science/hal-00501773}

Submitted on 12 Jul 2010

HAL is a multi-disciplinary open access archive for the deposit and dissemination of scientific research documents, whether they are published or not. The documents may come from teaching and research institutions in France or abroad, or from public or private research centers.
L'archive ouverte pluridisciplinaire HAL, est destinée au dépôt et à la diffusion de documents scientifiques de niveau recherche, publiés ou non, émanant des établissements d'enseignement et de recherche français ou étrangers, des laboratoires publics ou privés. 


\section{Author's Accepted Manuscript}

Excitation Symmetry Analysis Method (ESAM) for calculation of higher order nonlinearities

Serge Dos Santos, Camille Plag

PII:

DOI:

S0020-7462(07)00236-3

Reference:

doi:10.1016/j.ijnonlinmec.2007.12.014

NLM 1430

To appear in: International Journal of Non-

Linear Mechanics

Received date: 25 May 2007

Revised date: 20 November 2007

Accepted date: 18 December 2007
-

NON-LINEAR MECHANICS

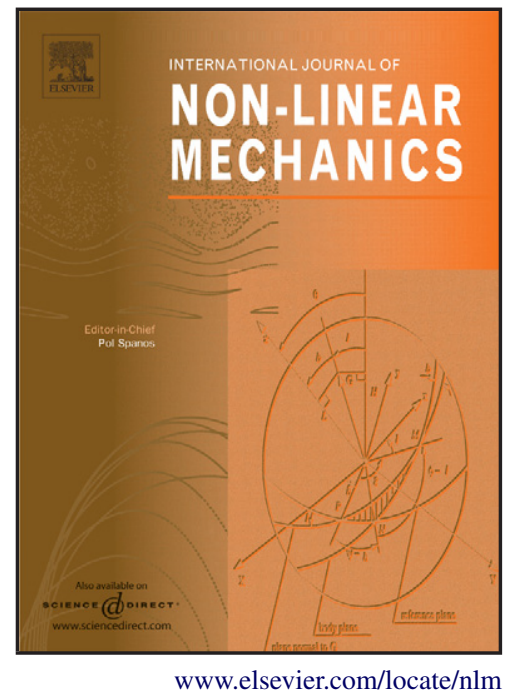

www.elsevier.com/locate/nlm

Cite this article as: Serge Dos Santos and Camille Plag, Excitation Symmetry Analysis Method (ESAM) for calculation of higher order nonlinearities, International Journal of Non-Linear Mechanics (2007), doi:10.1016/j.ijnonlinmec.2007.12.014

This is a PDF file of an unedited manuscript that has been accepted for publication. As a service to our customers we are providing this early version of the manuscript. The manuscript will undergo copyediting, typesetting, and review of the resulting galley proof before it is published in its final citable form. Please note that during the production process errors may be discovered which could affect the content, and all legal disclaimers that apply to the journal pertain. 


\title{
Excitation Symmetry Analysis Method (ESAM) for Calculation of Higher Order Nonlinearities
}

\author{
Serge Dos Santos and Camille Plag \\ ENI Val de Loire, Laboratoire Ultrasons Signaux et Instrumentation (LUSSI) de \\ l'Université François Rabelais FRE 2448 CNRS - GIP Ultrasons, \\ ENIVL, Rue de la Chocolaterie BP 3410, F-41034 BLOIS cedex, France
}

\begin{abstract}
A nonlinear system with third order nonlinearity is fully characterized using Symmetry Analysis applied to the excitation, as it is done in second order nonlinear systems using the pulse inverted method. Symmetry Analysis is performed using irreductible representations and the character table of $C_{3}$ rotation point group, which leads to the construction of three eigen-excitations allowing extraction of the third order nonlinearity parameter without the perturbation of fundamental and second order terms. Validation of this concept is based on Excitation Symmetry Analysis Method (ESAM) which was tested on simulated noisy signals and compared with classical spectral analysis.
\end{abstract}

Key words: elsart, document class, instructions for use PACS: 43.25-x, 43.25Ts,43.60Wy Nonlinear Systems, Symmetry Analysis, eigen-excitations

\section{Introduction}

The Pulse Inversion (PI) technique is one of the most successful signal processing tools in second harmonic imaging due to its ability to separate second order harmonic components regardless of the transmitted bandwidth[1]. The PI success is not only restricted to Doppler imaging where multiple pulses are transmitted whereby adjacent pulses are inverted relative to their neighboring

Email address: s.pepping@elsevier.com (Serge Dos Santos and Camille Plag). URL: authors.elsevier.com/locate/latex (Serge Dos Santos and Camille Plag). 
pulses, but also to any characterization in the time domain, of second order nonlinear systems. During the last two decades, higher order nonlinearities seem to bring additional information in various applications such as biomedical engineering, where signal contrast improvement on tissue is achieved with contrast agent[2], or non destructive testing[3], where nonclassical nonlinearity seems to be present.

The success of these new imaging systems has lead to the natural investigation of the use of higher harmonics and associated new signal processing tools. Nevertheless, most nonlinear systems, such as materials with cracks[4], exhibit third order nonlinearities when they are analyzed with ultrasound imaging, making it impossible to use the PI method. Various signal processing methods, including high order Fourier and wavelet analysis, which mix time and frequency domains have shown their interest in such systems. All of them use the concept of specific representations of signals in suitable spaces, which are intrinsically linked to symmetries and where invariants have to be extracted.

Furthermore, there is an increase in the use of Symmetry Analysis (SA) for nonlinear systems[5] due to its ability to simplify and sometimes completely solve the nonlinear problem under investigation (see for example [6,7] where practical applications of this method have been implemented). SA can be used as a powerful tool for defining specific transformations of space called "similarity transformations" of signals coming from nonlinear systems, on which classical signal processing can be performed.

In this paper, we define an extension of the PI method using the concept of Symmetry Analysis (SA). Group properties are used to define specific excitations called eigen-functions which allow characterization of the 3rd order nonlinearity. The objective of this paper is to interpret PI with the group theoretical point of view and use group analysis for the definition of its extension, called Excitation Symmetry Analysis Method (ESAM). We propose a quantitative comparison between results using ESAM and classical system analysis.

\section{SA interpretation of Classical PI method}

Let us consider a second order nonlinear system $(\mathcal{S})$ excited with $x(t)$, and where the response $y(t)$ is given by :

$$
y(t)=N L[x(t)]=N_{1} x(t)+N_{2} x^{2}(t),
$$

where $N_{1}$ and $N_{2}$ are respectively linear and second order coefficients. If $X_{E}=$ $x(t)$ and $X_{I}=-x(t)$ are applied separately to $(\mathcal{S})$, one can extract $N_{1}$ and 
$N_{2}$ using the respective nonlinear response $Y_{E}(t)$ and $Y_{I}(t)$ with :

$$
\begin{aligned}
& N_{1}=\frac{Y_{E}(t)-Y_{I}(t)}{2 x(t)}, \\
& N_{2}=\frac{Y_{E}(t)+Y_{I}(t)}{2 x^{2}(t)} .
\end{aligned}
$$

In classical PI, the use of Eq.(3) leads to the measurement of second order nonlinearity signature $N_{2}$ with high amplitude pulses, where adjacent pulsed excitations are applied to the nonlinear medium and imaged with classical Doppler instrumentation.

Table 1

\begin{tabular}{c|cc|c|cc}
$C_{2}$ & $E$ & $I$ & $C_{2}$ & $E$ & $I$ \\
\hline \hline$E$ & $E$ & $I$ & $A_{g}$ & 1 & 1 \\
$I$ & $I$ & $E$ & $A_{u}$ & 1 & -1 \\
\hline
\end{tabular}

Multiplication table (left) and character table (right) for the point group $C_{2}$. The top row of the right table labels the group elements, and the first column labels the one-dimensional irreducible even $A_{g}$ and odd $A_{u}$ representations. $E$ is the identity and $I$ denotes inversion or rotation by an angle $\pi$

In terms of symmetry properties[8], excitation $X_{E}$ and $X_{I}$ can be associated to neutral element $E$ and inversion element $I$ of the $C_{2}$ group of inversion whose properties are given in Table 1 . In fact, if we define $\Phi_{A_{u}}=Y_{E}(t)-Y_{I}(t)$ and $\Phi_{A_{g}}=Y_{E}(t)+Y_{I}(t)$ which appear respectively in Eq.(2) and Eq. (3), one can see that linear signature $N_{1}$ and nonlinear signature $N_{2}$ are completely isolated and extracted thanks to these functions. On the other hand, $\Phi_{A_{g}}=2 N_{2} x^{2}(t)$ and $\Phi_{A_{u}}=2 N_{1} x(t)$ can be seen as eigen-response of the system with respect to linear and nonlinear parts. Signatures $N_{1}$ and $N_{2}$ can be extracted from $\Phi_{A_{g}}$ and $\Phi_{A_{u}}$, assuming a normalization by the input $x(t)$ for $\Phi_{A_{u}}$ and $x^{2}(t)$ for $\Phi_{A_{g}}$. It is important to note that this calculation is not dependant of spectral content of excitation $x(t)$. Properties of $(\mathcal{S})$ with respect to group inversion $C_{2}$ allow the separation between linear and nonlinear parts. The concept of symmetrization of excitation is then defined if we consider that extraction of the nonlinear signature $N_{2}$ has been obtained thanks to the action of the group elements $E$ and $I$ acting on the excitation $x(t)$.

Assuming isomorphism properties between the inversion group and the rotation point group $C_{2}$, equivalent interpretation can be proposed in order to link our analysis to classical results concerning PI. If we consider the single frequency excitation $x(t)=x_{0} e^{j \omega t}$, where $\omega$ is the angular frequency, the action of inversion $I$ on excitation $x(t)$, ie $X_{I}$ can be written as :

$$
X_{I}=-x_{0} e^{j \omega t}=x_{0} e^{j \omega t} e^{-j \pi}=x_{0} e^{j \omega t-j \pi}=x_{0} e^{j(\omega t-\pi)},
$$


which is interpreted as a $\pi$-phase shift of the excitation $x(t)$. Consequently, the equivalence between the inversion group $C_{I}$ and the rotation group $C_{2}$ is again validated. This link between $C_{I}$ and $C_{2}$ groups can be also recovered if we try to find the rotation angle $\phi$ which must be applied to $x(t)$ and $x(t) e^{j \phi}$ in order to obtain Eqs.(2-3). Assuming $y_{\phi}=N L\left[x(t) e^{j \phi}\right]$, one obtains :

$$
\begin{aligned}
& y_{\phi}=N_{1} x(t) e^{j \phi}+N_{2} x^{2}(t) e^{2 j \phi}, \\
& y_{0}=N_{1} x(t)+N_{2} x^{2}(t),
\end{aligned}
$$

and then

$$
\begin{aligned}
& \Phi_{A_{g}}=y_{\phi}+y_{0}=N_{1}\left(1+e^{j \phi}\right) x(t)+N_{2}\left(1+e^{2 j \phi}\right) x^{2}(t), \\
& \Phi_{A_{u}}=y_{\phi}-y_{0}=N_{1}\left(1-e^{j \phi}\right) x(t)+N_{2}\left(1-e^{2 j \phi}\right) x^{2}(t) .
\end{aligned}
$$

In order to obtain uncoupled equations with respect to $N_{1}$ and $N_{2}, \phi=\pi$ can be chosen, and demonstrate that $C_{2}$ group properties simplify the problem.

\subsection{Generalization of Symmetrization of Excitation : ESAM Analysis}

Let us extend the previous analysis in order to consider a third order nonlinear system $(\mathcal{S})$ :

$$
y(t)=N L[x(t)]=N_{1} x(t)+N_{2} x^{2}(t)+N_{3} x^{3}(t),
$$

where $N_{3}$ is the third order coefficient. Let us apply the previous method using properties of the $C_{3}$ group for which properties (multiplication and character tables) are given in Table 2.

Table 2

\begin{tabular}{c|ccc|c|ccc}
$C_{3}$ & $E$ & $\epsilon$ & $\epsilon^{*}$ & $C_{3}$ & $E$ & $\epsilon$ & $\epsilon^{*}$ \\
\hline$E$ & $E$ & $\epsilon$ & $\epsilon^{*}$ & $A_{1}$ & 1 & 1 & 1 \\
$\epsilon$ & $\epsilon$ & $\epsilon^{*}$ & $E$ & $A_{2}$ & 1 & 1 & -1 \\
$\epsilon^{*}$ & $\epsilon^{*}$ & $E$ & $\epsilon$ & $E_{1}$ & 2 & -1 & 0
\end{tabular}

Multiplication table (left) and character table (right) for the point group $C_{3} . E$ is the identity, $\epsilon=e^{\frac{2 i \pi}{3}}$ denotes rotation by an angle $\frac{2 \pi}{3}, \epsilon^{*}=e^{-\frac{2 i \pi}{3}}$ denotes rotation by an angle $-\frac{2 \pi}{3}$. The first column of the right table labels the irreducible representations $\left(A_{1}, A_{2}, E_{1}\right)$ and the top row labels the group elements $\left(E, \epsilon, \epsilon^{*}\right)$

The basic result of group theory states that basis functions of irreducible representations of discrete groups can be obtained by using irreducible character 
table[8]. Generalization of the previous method can be done using the transformation operator $U_{g}$ associated to group element $g$, acting on the initial excitation $x(t)$ in order to built additional excitation $X_{g}$ :

$$
\begin{aligned}
& X_{E}=U_{E}(x(t))=x(t), \\
& X_{\epsilon}=U_{\epsilon}(x(t))=x(t) e^{\frac{2 i \pi}{3}}, \\
& X_{\epsilon^{*}}=U_{\epsilon^{*}}(x(t))=x(t) e^{-\frac{2 i \pi}{3}} .
\end{aligned}
$$

As done previously for PI, the second step is to define eigen-responses $\Phi_{m}$ using $C_{3}$ group properties given in Tab.2, which can be associated to basis functions of irreducible representations $m$ :

$$
\begin{aligned}
& \Phi_{A_{1}}=\sum_{g \in C_{3}} \chi_{A_{1}}(g) Y_{g}, \\
& \Phi_{A_{2}}=\sum_{g \in C_{3}} \chi_{A_{2}}(g) Y_{g}, \\
& \Phi_{E}=\sum_{g \in C_{3}} \chi_{E}(g) Y_{g},
\end{aligned}
$$

where $\chi_{m}(g)$ is the character associated to group element $g$ of the $m$ th irreducible representation (Table 2), and $Y_{g}=N L\left[X_{g}\right]$ is the nonlinear response of $\mathcal{S}$ related to the excitation $X_{g}$ given by Eqs.(10-12). Starting from Eqs.(13-15), eigen-responses $\Phi_{m}$ are then given by :

$$
\begin{aligned}
& \Phi_{A_{1}}=1 Y_{E}+1 Y_{\epsilon}+1 Y_{\epsilon^{*}}, \\
& \Phi_{A_{2}}=1 Y_{E}+1 Y_{\epsilon}-1 Y_{\epsilon^{*},} \\
& \Phi_{E}=2 Y_{E}-1 Y_{\epsilon} .
\end{aligned}
$$

For $\Phi_{A_{1}}$ and after inserting nonlinear response $Y_{g}=N L\left[X_{g}\right]$, it yields :

$$
\begin{aligned}
\Phi_{A_{1}} & =N_{1} x(t)+N_{2} x^{2}(t)+N_{3} x^{3}(t) \\
& +N_{1} x(t) e^{\frac{2 i \pi}{3}}+N_{2} x^{2}(t) e^{\frac{4 i \pi}{3}}+N_{3} x^{3}(t) e^{\frac{6 i \pi}{3}} \\
& +N_{1} x(t) e^{\frac{-2 i \pi}{3}}+N_{2} x^{2}(t) e^{\frac{-4 i \pi}{3}}+N_{3} x^{3}(t) e^{\frac{-6 i \pi}{3}},
\end{aligned}
$$

which gives finally

$$
\Phi_{A_{1}}=\left(N_{1} x(t)+N_{2} x^{2}(t)\right)\left[1+e^{\frac{2 i \pi}{3}}+e^{\frac{-2 i \pi}{3}}\right]+3 N_{3} x^{3}(t) .
$$


For all eigen-responses, one finally obtains:

$$
\begin{aligned}
& \Phi_{A_{1}}=3 N_{3} x^{3}(t), \\
& \Phi_{A_{2}}=N_{3} x^{3}(t)-2 \epsilon\left[N_{2} x^{2}(t)+\epsilon N_{1} x(t)\right], \\
& \Phi_{E}=N_{3} x^{3}(t)+\left(2-\epsilon^{*}\right) N_{2} x^{2}(t)+(2-\epsilon) N_{1} x(t) .
\end{aligned}
$$

The most interesting application of this method is obtained using Eq.(21), related to the completely symmetric irreducible representation $A_{1}$, and which yields the extraction of the third harmonic component $N_{3}$ without any perturbation of $N_{1}$ and $N_{2}$ components. Furthermore, it can be shown that eigenresponse $\Phi_{A_{1}}$ can cancel all $p=3 n+1$ and $p=3 n+2$ coefficients of a general nonlinear system $\mathcal{S}$ where $N L[x(t)]=\sum_{p} N_{p} x^{p}$ and $\Phi_{A_{1}}=3 \sum_{i} N_{3 i} x^{3 i}$, which contain only $3 i$ terms. Generalization of the group procedure can be done using $C_{4}, C_{5}, \ldots$ point group symmetries and also properties of group products $C_{i} \times C_{j}$ for multiple inputs of the kind $x(t)=x_{1}(t)+x_{2}(t)$. All properties related to irreducible representations of direct products could be used to find new eigen-excitations, and constitutes an interesting practical perspective of this method.

\section{Practical evaluation of ESAM sensitivity}

Numerical simulations, done with Matlab software, have been performed in order to compare ESAM sensitivity with respect to classical Fourier Analysis, done with FFT. The objective is to compare the detection of the third harmonic generated by $S$ when one applies a classical tone-burst excitation $x(t)=\Pi_{\tau}(t) \cos \left(2 \pi f_{0} t\right)+\gamma(t)$, where $\Pi_{\tau}(t)$ is the window function of duration $\tau=90 \mu \mathrm{s}, f_{0}=1 \mathrm{MHz}$ is the frequency, $\gamma(t)$ a uniformly distributed white noise in the $[-b ; b]$ range. Comparison consists of evaluating the FFT amplitude of direct output response $y(t)$ and ESAM eigen-response $\Phi_{A_{1}}$ given by Eq.(13). A toneburst input signal $x(t)$ was chosen because of its interest in most nonlinear acoustics experiments applied to medical ultrasound and nondestructive testing. The choice of classical FFT is arbitrary and any signal processing tool can be applied without loss of generality.

As shown in Fig.1, the FFT amplitude of nonlinear response $y(t)$ (direct output response) presents classical behavior related to the presence of 2 nd and 3rd order nonlinearity : frequency lines at 2 and $3 \mathrm{MHz}$. Note that in the noiseless case $(b=0)$, with $N_{1}=10, N_{2}=0.158$ and $N_{3}=0.0348$, the 3 rd harmonic detection seems to be difficult to detect with the direct output response $y(t)$ whereas it is clearly present with ESAM eigen-response $\Phi_{A_{1}}$. As expected, the 


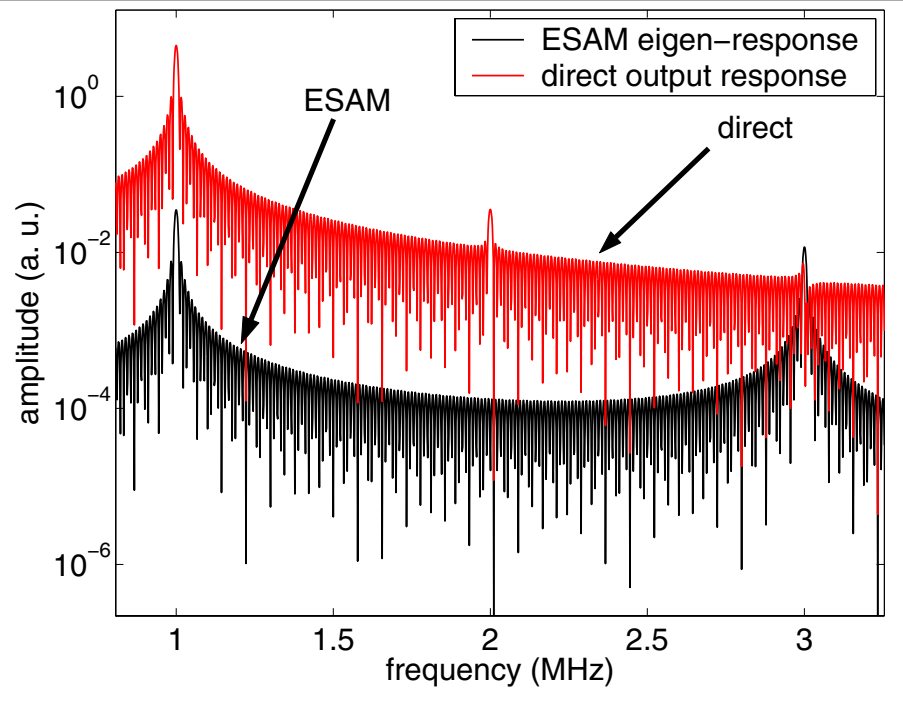

Fig. 1. Sensitivity comparison between direct output response $y(t)$ and eigen-reponse $\Phi_{A_{1}}$ extracted with ESAM. Parameters of the nonlinear system $\mathcal{S}$ are $: N_{1}=10$; $N_{2}=0.158 ; N_{3}=0.0348$.

third harmonic component is revealed, even if there is still consequences due to the FFT windowing, where classical ponderation windows like Hanning leading to suppression of these effects have not been used intentionally. In the particular case of Fig.1, ESAM dynamics of 3rd harmonic detection is evaluated to $20 \mathrm{~dB}$ whereas direct output response dynamic is of the order of $1 \mathrm{~dB}$. Detection of the fundamental and second harmonic is possible with $y(t)$ but difficult for the third harmonic, whereas ESAM dynamic for this third harmonic is about $20 \mathrm{~dB}$.

In order to evaluate ESAM threshold of sensitivity detection in the noiseless case $(b=0)$, the 3 rd order nonlinearity level $N_{3}$ has been decreased, keeping $N_{1}=10$ and $N_{2}=0.158$ constant. For each values of applied third order level $N_{3}$, the 3rd harmonic line amplitude level at $f_{3}=3 \mathrm{MHz}$ is evaluated using FFT.

Comparison of performances of the 3rd order harmonic detection is presented in Fig.2, where the measured 3rd order level at $f_{3}=3 \mathrm{MHz}$ has been done with direct output response $y(t)$ and with ESAM eigen-response $\Phi_{A_{1}}$ for various values of applied third order level of nonlinearity $N_{3}$. As expected, both methods allow the same evaluation of nonlinearity $N_{3}$ in the range $\left[10^{-2} ; 10^{2}\right]$. Arrow indicates points that have been calculated using the spectrum presented in Fig. 1 where $N_{3}=0.0348$. Threshold characterized by the plateau in the range $\left[10^{-7} ; 10^{-2}\right]$ of direct output response is about $410^{-3}$ given for $N_{3} / N_{1}=10^{-2}$.

Detection of nonlinearity $N_{3}$ is limited if we consider direct output response $y(t)$. Detection is no longer possible if $\frac{N_{3}}{N_{1}}=10^{-2}$ whereas ESAM detection is still possible for $\frac{N_{3}}{N_{1}}=10^{-7}$. Below $10^{-7}$, there is no interest of comparison of 
ACCEPTED MANUSCRIPT

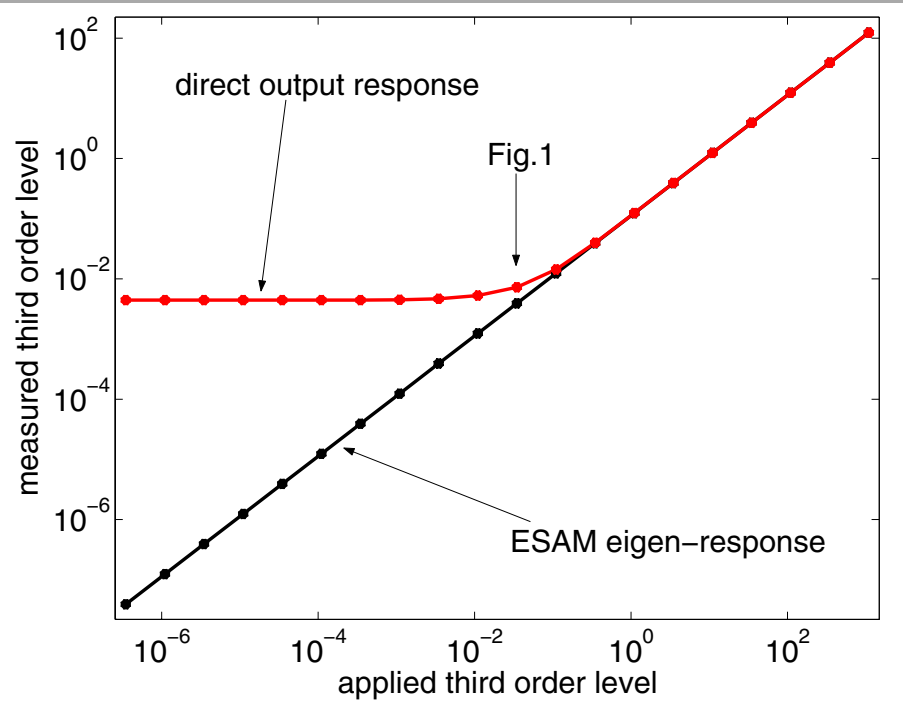

Fig. 2. ESAM sensitivity between eigen-response $\Phi_{A_{1}}$ and direct output response $y(t)$. Parameters are $: N_{1}=10 ; N_{2}=0.158 ; N_{3} \in\left[1.110^{3} ; 310^{-7}\right]$.

methods because of the lack of practical reality.

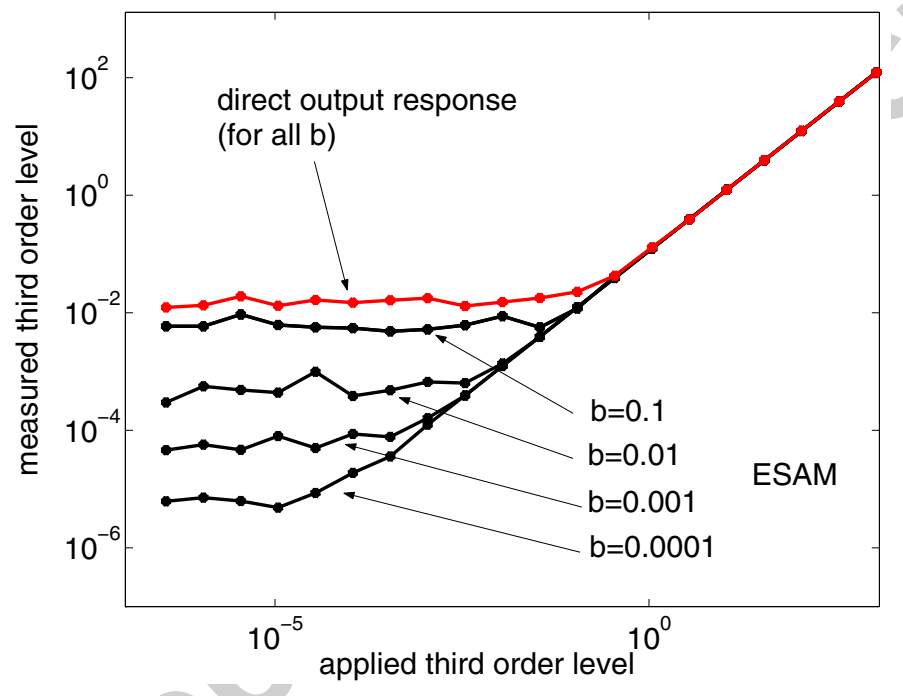

Fig. 3. ESAM Sensitivity between eigen-response $\Phi_{A_{1}}$ and direct output response $y(t)$ with noise level $b$.

Conversely, ESAM sensitivity has also been evaluated introducing noise $(b \neq$ 0 ) at each step of the Matlab calculations (Eqs.(9-13)) used for the simulations. Comparison between direct output and ESAM responses has been done for various values of $b$ (Fig.3). As expected, the ESAM sensitivity threshold increases as the noise level increases, but is always kept lower than the direct output sensitivity threshold. For $b=0.1$, ESAM sensitivity seems to reach a common limit as observed for direct output response. Exept for $b=0.1$ where noise effects are greater than FFT windowing ones, direct output response is not sensitive to noise variation, whereas ESAM presents a significant depen- 
dance $\left(10^{-4}\right.$ for $b=0.001 ; 10^{-2}$ for $\left.b=0.1\right)$. Note that the ESAM threshold increase from $N_{3}^{(\text {meas })}=510^{-5}$ to $10^{-2}$ for $b=10^{-1}$, whereas no variation is observed for direct output response measurements. This dependance can be explained by the fact that adding noise in the system can be interpreted as symmetry breaking. Eigen-responses $\Phi_{A}$, given by Eq.(21) is no longer proportional to $N_{3}$ and contains an additional term proportional to the noise level which becomes significant in the case of decreasing the value of $N_{3}$, or increasing the value of noise perturbation.

\subsection{ESAM test on real signals}

ESAM has been tested with real data coming from experiments in ultrasound imaging using TR-NEWS and NEWS-TR methods $[9,10]$ which are based on Nonlinear Elastic Wave Spectroscopy (NEWS) combined with Time Reversal Acoustics (TR). The main objective of this kind of experiments[11] is to combine TR-NEWS and PI in order to evaluate nonlinear properties of damaged steel samples with cracks. In practice, the first response (Fig.4) coming from ultrasonic wave propagation inside the sample is time reversed, used as a new excitation $x_{e}(t)$ and reinjected in the nonlinear medium. This $1 \mathrm{~ms}$ coda which contains several echoes coming from multiple reflections inside the sample, is time reversed and used as a new excitation in most of our experimental studies. Note that the experimental noise level amplitude is evaluated at $b=0.003$. Note that $x_{e}(t)$ intrinsic noise level has been evaluated on the first part of the signal $(t \in[0 ; 0.1] \mathrm{ms})$ to be a uniformly distributed white noise in the $[-b ; b]$ range, with $b=0.003$. The spectrum, presented in (b) reveals 3rd order harmonic line at $f=2.1 \mathrm{MHz}$ coming from intrinsic nonlinearity of the experimental set-up used for signal acquisition.

This new TR signal can have a rich spectral content, or can be polluted by spurious spectral lines coming from nonlinearity, or can have been filtered by bandwidth of the system. In any case, thanks to ESAM normalisation process intrinsically contained (see Eq.(2) for example), theses effects will have no incidence to the quality of $N_{1}$ and $N_{2}$ evaluation. ESAM sensitivity evaluation has been done with this experimental signal as it was done previously on the tone-burst.

As shown in Fig.4(a), initial signal $x_{e}(t)$ contains several tone-bursts at $f_{0}=$ $700 \mathrm{kHz}$ coming from wave reflections inside the sample, and also nonlinearity (spectral line at $3 f_{0}=2.1 \mathrm{MHz}$ ) in Fig.4(b), where its origin may come from acoustic propagation, electronics or signal quantification. Note that $x_{e}(t)$ intrinsic noise level has been evaluated on the first part of the signal $(t \in$ $[0 ; 0.1] \mathrm{ms})$ to be a uniformly distributed white noise in the $[-b ; b]$ range, with $b=0.003$. The noise value $(b=0.003)$ was added inside the ESAM sensitivity 


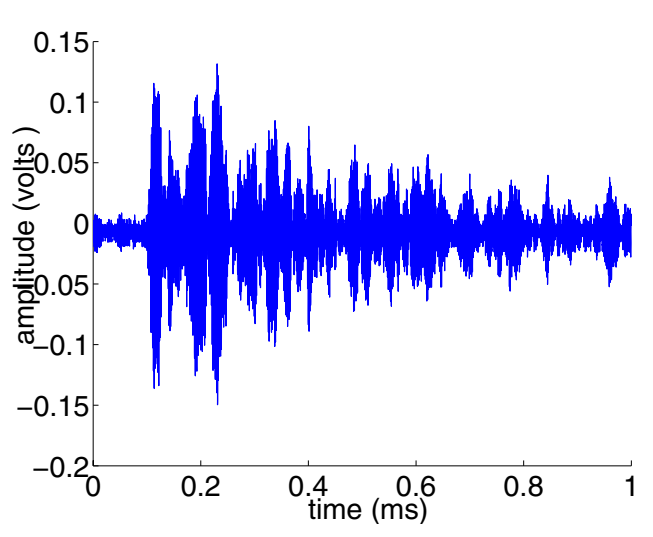

(a) initial excitation

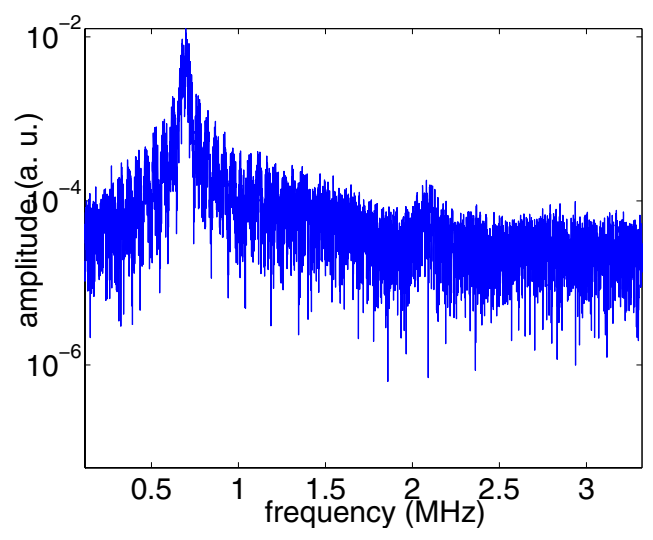

(b) spectrum

Fig. 4. Properties of the experimental signal $x_{e}(t)$ in the time (a) and frequency (b) domains, coming from the measurement of ultrasonic tone-burst propagation at $f=700 \mathrm{kHz}$ in a steel sample with dimensions $1.2 \times 2.5 \times 10 \mathrm{~cm}$.

evaluation with $x_{e}(t)$ as input signal. As previously done, ESAM sensitivity is compared to the direct output response for $N_{3}$ evaluation using $x_{e}(t)$ as the initial excitation. The results are presented in Fig. 5 where threshold difference between ESAM and direct output response is about $10 \mathrm{~dB}$. It can be noticed that ESAM sensitivity does not depend on the presence of nonlinearity in the initial excitation where, in the latter, a harmonic low pass digital filter with a cutoff frequency $f_{c}=1.4 \mathrm{MHz}$ has been applied to $x_{e}(t)$ before its use as excitation. In the first case of evaluation of nonlinearity with direct output response, there is a bias due to the presence of component of the third order in the initial excitation $x_{e}(t)$. Conversely, it is shown here that ESAM does not depend on the spectral component of initial excitations $x_{e}(t)$ whereas classical analysis based on 3rd harmonic generation is clearly dependant on the initial 3rd harmonic level. ESAM sensitivity threshold is about $7 \mathrm{~dB}$ higher than classical analysis using the spectrum of $y_{e}(t)=N L\left[x_{e}(t)\right]$ even if the harmonic filtering at $f_{c}=1.4 \mathrm{MHz}$ has been applied in order to eliminate the intrinsic 3rd order harmonic nonlinearity of $x_{e}(t)$. As expected, direct output response has a better sensitivity due to the suppression of intrinsic nonlinearity, where ESAM presents the same sensitivity level, showing its non-dependance on the harmonic presence in the initial excitation.

\section{Conclusion}

Analysis of the third order nonlinear systems has been conducted using a new method which consists of Symmetry Analysis of Excitations (ESAM). Thanks to the group theoretical approach, eigen-excitations have been constructed 
ACCEPTED MANUSCRIPT

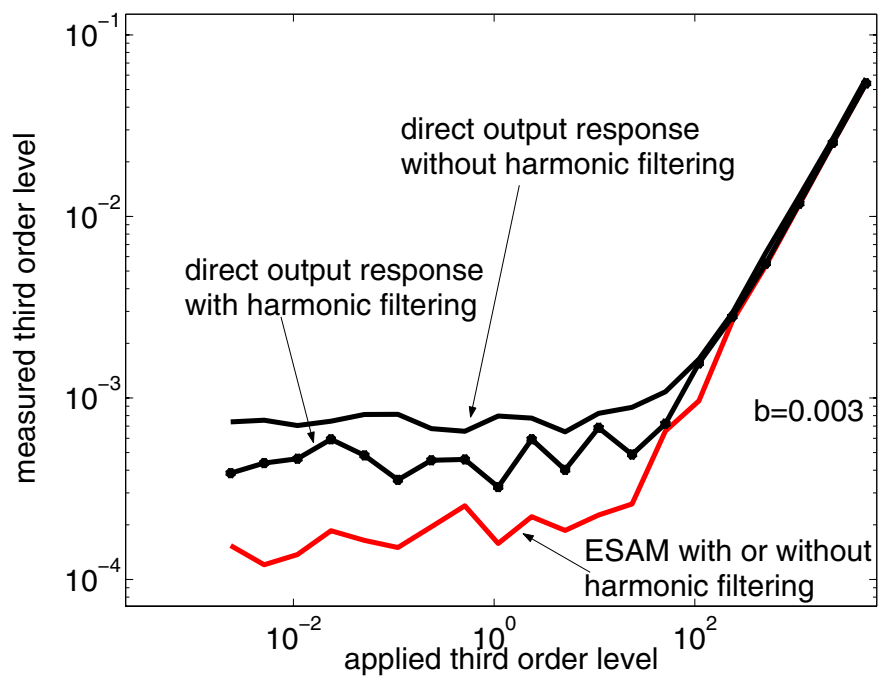

Fig. 5. Comparison between ESAM and direct output response sensitivity for $N_{3}$ third order nonlinearity evaluation, using a real signal $x_{e}(t)$ given by Fig. 4 and where added noise level is $b=0.003$.

and applied to the nonlinear system $\mathcal{S}$ leading to the extraction of the third order harmonic component alone. It is done using intrinsic symmetries of $\mathcal{S}$ independently of properties of initial excitation applied to $\mathcal{S}$. Irreducible representation and character tables properties of $C_{3}$ point group of rotation are on the heart of the analysis. ESAM sensitivity has been compared to classical analysis in noisy systems and with experimental data. The main result is the power of this technique which can be easily extended to higher order analysis. Another interesting result is that ESAM is not sensitive to the presence of spurious nonlinearity, leading to a suitable method for nonlinearity evaluation of the system under consideration. A high order extension of ESAM will be conducted with odd nonlinearities coming from Nonclassical Nonlinearity generated in hysteretic systems[3]. As a perspective, practical implementation of ESAM could be proposed for exciting a medium with odd nonlinearities: damaged material with cracks and bubbly biological medium where superharmonics responses could be extracted with an extension of Harmonic Imaging using the PI method.

\section{References}

[1] D. Hope Simpson, C. Chin, P. Burns, Pulse invertion Doppler : a new method for detecting nonlinear echoes from microbubble contrast agents, IEEE Trans. Ultrason. Ferr. Freq. Control, 46, no 2, pp.372-382, 2002.

[2] B. Haider, R. Chiao, Higher order nonlinear ultrasonic imaging, in Proceedings of the 1999 IEEE Ultrasonics Symposium, pp. 3510-3517 
[3] L. A. Ostrovsky and P. A. Johnson, "Dynamic nonlinear elasticity of geomaterials," La Rivista del Nuovo Cimento, vol. 24, pp. 1-46, 2001.

[4] I.Yu. Solodov, N. Krohn and G. Busse, CAN: an example of nonclassical acoustic nonlinearity in solids, Ultrasonics, 40, (2002).

[5] B. J. Cantwell, Introduction to Symmetry Analysis, New York: Cambridge University Press, 2002.

[6] S. Dos Santos, Symmetry of nonlinear acoustics equations using group theoretic methods : a signal processing tool for extracting judicious physical variables, in Proceedings of the Joint Congress CFA/DAGA'04, Strasbourg, 2004, pp. 549550 .

[7] S. Dos Santos, V. Gusev, L. Haumesser, F. Vander Meulen and O. Bou Matar, Optimized Excitation Sources and Velocity Exact Solutions for Ultrasonic Field Propagation in a Nonlinear Medium With Hysteretic Behavior, in Proceedings of the IEEE International Ultrasonics, Ferroelectrics, and Frequency Control, 2004

[8] M. Tinkham, Group Theory and Quantum Mechanics, New York: McGraw-Hill, 1964.

[9] T. Goursolle, S. Calle, S. Dos Santos, and O. Bou Matar, A two-dimensional pseudospectral model for time reversal and nonlinear elastic wave spectroscopy,J. Acoust. Soc. Am., 122 (6), (2007)

[10] Thomas Goursolle, Serge Dos Santos, Olivier Bou Matar and Samuel Calle, NonLinear based Time Reversal Acoustic applied to crack detection: Simulations and Experiments, submitted to International Journal of Non-Linear Mechanics.

[11] P.-Y. Le Bas, K.E. Van Den Abeele, S. Dos Santos, T. Goursolle, O. Bou Matar, Experimental Analysis for Nonlinear Time Reversal Imaging of Damaged Materials, Proc. of European Congress of NDT, sept 25-29 (2006) 